\title{
Em Busca de Perfis Cognitivos de Alunos em Blended Learning
}

\author{
Lucas Viana, Thaís Castro, Bruno Gadelha \\ Instituto de Computação - Universidade Federal do Amazonas (UFAM) \\ Av. Gen. Rodrigo Otávio, Coroado - Manaus - AM - Brazil \\ \{lucaseicomp.ufam.edu.br, thaiseicomp.ufam.edu.br, \\ brunodicomp.ufam.edu.br
}

\begin{abstract}
Teachers who teach courses in blended learning using LMS have difficulties keeping track of all students due to their lack of knowledge about students' cognitive characteristics. This article proposes a way of identifying cognitive profiles of students, based on a detailed analysis of the activities proposed and performed in an AVA referring to a course taught in the blended learning for undergraduate majors of different areas of knowledge. This classification, resulting from the proposed cognitive modeling, will be implemented through students' clustering during their interaction in the course.

Resumo. Professores de disciplinas na modalidade semipresencial apoiadas pelo uso de AVA possuem dificuldades em acompanhar adequadamente os alunos por não perceberem as suas características cognitivas. Neste artigo é proposta uma maneira de identificação de perfis cognitivos de alunos, com base em uma análise detalhada das atividades propostas e realizadas em um AVA referente a uma disciplina ministrada na modalidade semipresencial para cursos de graduação de diferentes áreas do conhecimento. Essa classificação, resultante da modelagem cognitiva proposta, será implementada através de perfis individualizados que serão ajustados conforme clusterização dos alunos durante as interações realizadas na disciplina.
\end{abstract}

\section{Introdução}

Com o passar dos anos as tecnologias vem transformando e inovando cada vez mais o ambiente do ser humano. Uma das transformações e evoluções se situa no âmbito educacional com a adoção da aprendizagem em blended learning, método que utiliza aulas presenciais e aulas em ambiente virtuais [Porter et al., 2014; Santo e Oliveira 2011]. As vantagens pedagógicas proporcionadas pela aprendizagem em blended learning são o aumento da eficácia, satisfação e eficiência da aprendizagem [Graham, 2013]. Além disso, o professor pode utilizar o Ambiente Virtual de Aprendizagem (AVA) como repositório de arquivos dos conteúdos ensinados em sala de aula e determinar exercícios, exames, tarefas e etc. para serem realizados [Allen \& Seaman, 2013]. Um AVA na modalidade semipresencial fornece flexibilidade, porém requer que os alunos participem de aulas presenciais quando estas forem programadas, de forma que consiga se organizar melhor para as atividades a distância. Pelo lado do professor, possibilita uma troca maior entre os alunos de diferentes turmas, uma vez que organize 
VII Congresso Brasileiro de Informática na Educação (CBIE 2018)

Anais do XXIX Simpósio Brasileiro de Informática na Educação (SBIE 2018)

o ambiente, adaptando os conteúdos didáticos e estruturando as atividades para a realidade dos alunos [Aldhafeeri, 2015; Artino e Jones, 2012].

As aulas virtuais podem ser feitas em horários flexíveis, proporcionando ao aluno melhor gerenciamento dos seus horários. Dessa forma, o aluno pode aproveitar melhor seu tempo com outras prioridades como trabalho e família. Porém, tudo isso é conquistado a um custo, pois a oportunidade de se estar presencialmente com o professor e com os colegas é reduzida. Neste tipo de aprendizagem o aluno precisa saber o quanto é importante o conhecimento a ser adquirido na disciplina e determinar quando e como se envolver com o conteúdo da disciplina [Kizilcec, Pérez-Sanagustín e Maldonado, 2017; Moore \& Kearsley, 2005].

Essa modalidade semipresencial com utilização de blended learning pode também proporcionar um acompanhamento mais profundo e individualizado através da identificação de características comuns entre grupos de alunos que os levam a desistir da disciplina, a não realizar atividades de leitura, a entregar atividades sem reflexão e outras. Conforme o aluno realiza o gerenciamento de seus horários, se envolve e interage com seus pares e com o material na disciplina, pode ser formado um perfil cognitivo daquele aluno com base nessas suas características, possibilitando a intervenção automática do AVA, ou repassando alertas ao professor para agir ou com ações pré-programadas como oferta de material e atividade diferenciados.

Neste artigo, será explorada a identificação de perfis cognitivos iniciais emergentes de um estudo de caso observacional, com observação participativa em três turmas da disciplina Informática Instrumental da UFAM. Para isso, na Seção 2 são abordados alguns trabalhos correlatos, formando o contexto teórico metodológico dessa pesquisa. A Seção 3 descreve especificamente o método utilizado e o locus da pesquisa. Já na Seção 4 são apresentados resultados do estudo de caso e a Seção 5 apresenta uma discussão sobre os dados encontrados.

\section{Clusters e Perfis Cognitivos}

Na pesquisa de Vanslambrouck et al. (2015) para formação de perfis ou clusters, é analisado o histórico do aluno com relação a educação a online, sua situação sociodemográfica e socioeconômica e suas características psicológicas, como a motivação para aprender. Para tratar a situação sociodemográfica e socioeconômica os autores analisaram as variáveis de idade, gênero, escolaridade, profissão e estado civil. Com relação à motivação, foi utilizada a escala de motivação acadêmica de Vallerand et al (1992). Na análise dos dados foram encontrados três tipos de clusters, e essa caracterização para cada clusters seguiu como base a pesquisa de Ratelle et al [2007].

Outros autores como Watson et al (2017) investigavam os perfis cognitivos dos alunos dentro dos Massive Open Online Course (MOOC). Esses ambientes se caracterizam por conterem cursos destinados a uma grande quantidade de pessoas, o que faz emergir outra característica desse ambiente, que é a dificuldade em tratar a individualidade de cada aluno [Beaven, Codreanu e Creuze, 2014]. Como meio de entender melhor os alunos, os pesquisadores analisaram os comportamentos dos alunos 
VII Congresso Brasileiro de Informática na Educação (CBIE 2018)

Anais do XXIX Simpósio Brasileiro de Informática na Educação (SBIE 2018)

e suas necessidades individuais. Nesse trabalho, a categorização dos comportamentos foi analisada com base nos padrões comportamentais de Balakrishnan e Coetzee (2013) e as necessidades individuais foram analisadas através das seguintes variáveis: gênero, objetivo da inscrição no curso, acesso a avaliação em vídeo e satisfação, seguindo a linha do estudo feito por Kizilcec, Piech e Schneider (2013). Além disso, foram analisados outros aspectos particulares dos alunos como preferência por tipo de material didático do curso e preferências de design do ambiente. Após a análise dos dados foram observados dois perfis, os quais diferenciaram na satisfação dos alunos quantos ao MOOC. Os perfis encontrados foram: pessoas satisfeitas ou ficando satisfeitas com o ensino do MOOC e pessoas insatisfeitas e em declínio.

Além de Watson outros pesquisadores também vêm estudando a formação de perfis cognitivos, como é o caso de Vanslambroucka et al. (2018) que investigou os perfis em um curso de ensino superior ministrado em AVA. Para formação dos clusters foram consideradas as seguintes variáveis: idade, sexo, motivação para aprender ( a motivação para aprender foi medida utilizando o método Academic Motivation Scale (AMS) de Vallerand et al. (1992) e dados capturados a partir de uma entrevista com perguntas sobre a experiência de vida dos alunos, razões pelas quais foi escolhido o ambiente online e qual o valor dado por eles na educação do ambiente (online e combinada). $\mathrm{Na}$ análise foram categorizados os resultados conforme a teoria do valor de expectativa [Wigfield \& Eccles, 2000], com base nisso os perfis cognitivos foram categorizados em: valor intrínseco, valor de realização, valor de utilidade e custos.

Em termos de auto regulação na aprendizagem em blended learning, Broadbend (2017) define os perfis cognitivos dos alunos quando às estratégias adotadas por eles para se autorregularem e conseguirem acompanhar as disciplinas. Baseado nessas estratégias ele encontra e enumera os caminhos seguidos pelos alunos com relação a essas estratégias, que podem ser cognitivas, que tratam da organização do conteúdo e preparação das atividades; metacognitivas correspondendo às reflexões acerca dos tópicos da disciplina; e de gerenciamento de recursos, relacionadas a tempo, regulação do esforço, aprendizagem por pares e busca de ajuda. O desejável é que os alunos tenham um perfil de gerenciamento de recursos no início e depois migrem para perfis cognitivos e metacognitivos, não se fixando em somente um perfil.

Neste contexto essa pesquisa identifica perfis cognitivos relacionados ao trabalho descrito em [Beaven, Codreanu e Creuze, 2014] e [Balakrishnan e Coetzee 2013] os quais identificam padrões comportamentais. Os perfis cognitivos identificados neste trabalho são ainda iniciais e foram encontrados na disciplina de Informática Instrumental ministrada para cursos de graduação na modalidade semipresencial, utilizando blended learning, em comparação aos outros estudos esta pesquisa além de analisar os dados gerados pelo AVA também considera na análise o estudo observacional feito dentro da sala de aula e a partir disso encontrar soluções pedagógicas para o melhor engajamento do aluno. 
VII Congresso Brasileiro de Informática na Educação (CBIE 2018)

Anais do XXIX Simpósio Brasileiro de Informática na Educação (SBIE 2018)

. Essa disciplina faz parte da grade curricular de vários cursos de graduação, cursos esses das áreas de Ciências Exatas, Humanas e Biológicas. Na Universidade Federal do Amazonas (UFAM), a disciplina Informática Instrumental visa instrumentar os alunos, que não são de computação, nas tecnologias de informação e comunicação para que eles possam utilizar a tecnologia para melhor desempenhar suas atividades acadêmicas e profissionais.

\section{Método}

Essa pesquisa utilizou a metodologia de estudo de caso do tipo exploratório e observacional, segundo [Yin 1994], para analisar o comportamento de alunos através da observação participante, questionários e os dados de acesso, logs, de uma disciplina de informática básica ministrada para cursos de graduação da UFAM. A formatação e organização dos dados gerou clusters após o cruzamento das informações dos logs de acesso, do material didático e da resolução das atividades, possibilitando os indícios de identificação dos perfis cognitivos encontrados.

Como metodologia também, parte do estudo de caso, está o acompanhamento minucioso de três turmas da disciplina Informática Instrumental, contendo um total de 164 alunos de três cursos de diferentes áreas, Ciências Contábeis, Engenharia Civil e Engenharia de Alimentos, possuindo como suporte três professores, um tutor presencial e dois tutores a distância.

As aulas ocorrem duas vezes por semana, uma aula teórica, e outra prática ou até duas aulas práticas na mesma semana, dependendo da necessidade de ajuste do curso de graduação a quem se destina. As aulas teóricas são os dias que os professores estão dentro de sala de aula explicando o conteúdo e tirando dúvida dos alunos, já as aulas práticas são acompanhadas pelos tutores, que realizam supervisão na resolução de exercícios dos alunos, porém como a disciplina é semipresencial a aula prática pode ser realizada fora das dependências da UFAM. Todavia o aluno deve acessar o ambiente do moodle, para realizar os exercícios propostos pelos professores e garantir a sua presença. Caso o aluno sinta alguma dificuldade na resolução dos exercícios e esteja fora das dependências do campus, podem ser consultados um dos tutores ou professor pelo módulo de mensagens do moodle. Nas aulas práticas os monitores estão online para um retorno rápido ao aluno.

Os conteúdos no moodle foram agrupados em unidades, essas unidades são mostradas para os alunos conforme o progresso da disciplina. Conforme a Figura 1, cada unidade conta com vários recursos didáticos sendo: vídeos, tutoriais e slides e claro as atividades e os exercícios propostos pelos professores. O projeto pedagógico da disciplina evidencia como objetivo tornar o aluno autônomo na utilização das ferramentas de informática, na resolução de problemas em geral e no trabalho colaborativo [Silva et al. 2011]. 
VII Congresso Brasileiro de Informática na Educação (CBIE 2018)

Anais do XXIX Simpósio Brasileiro de Informática na Educação (SBIE 2018)

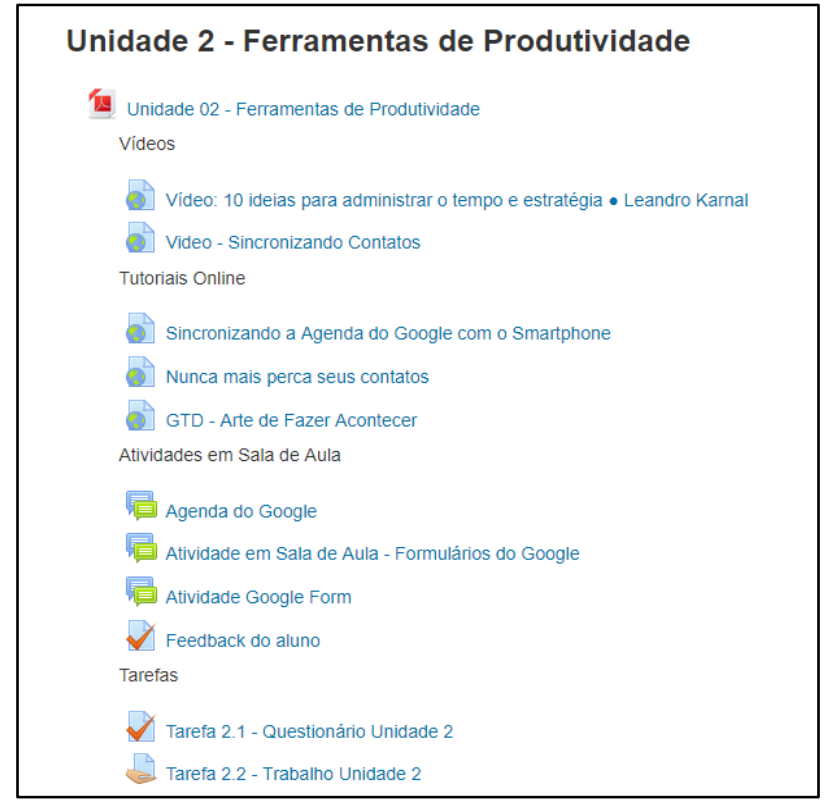

Figura 1. Unidade 2 da disciplina Informática Instrumental.

\section{Perfis Cognitivos e Análise dos Dados}

O foco da pesquisa é no estudo de variáveis relacionadas ao comportamento dos alunos no ambiente virtual de aprendizagem (AVA) que possibilitem a categorização do mesmo. As categorias encontradas correspondem a um perfil cognitivo inicial, baseado no comportamento dos alunos durante a disciplina.

Para formação dos clusters foi considerado o comportamento dos alunos no AVA com relação à resolução das atividades e visualização do conteúdo didático durante todo o período letivo. Para essa análise, as variáveis utilizadas foram: data e hora da resolução das atividades, data e hora da visualização do conteúdo didático, quantidade de visualizações do conteúdo didático e quantidade de acesso no curso. Tais informações encontram-se registradas em arquivos $(\log s)$ distintos no AVA utilizado e foram combinadas a fim de possibilitar a identificação de comportamentos comuns dos alunos.

Dessa forma, foi possível observar dois perfis comportamentais como parte da formação dos perfis cognitivos, retirados do modelo de estratégias cognitivas e metacognitivas, definido por [Broadbent 2017] e outros dois perfis de alunos que não produzem dados suficientes no AVA para qualificar suas atividades cognitivas, mas ainda assim se caracterizam como dois clusters distintos:

1. Gerente - é o aluno que independente das atividades nas outras disciplinas, entrega no prazo os exercícios e as atividades e visualiza o conteúdo previamente. Segundo o modelo, está situado no grupo das estratégias de gerenciamento de recursos, com duas das 4 habilidades, gerenciamento do tempo e regulação de esforço. 
VII Congresso Brasileiro de Informática na Educação (CBIE 2018)

Anais do XXIX Simpósio Brasileiro de Informática na Educação (SBIE 2018)

2. Inconsistente - é o aluno que visualiza a maioria do conteúdo previamente e entrega muitos exercícios, mas em alguns momentos não visualiza o conteúdo ou deixa de fazer alguma atividade. Segundo o modelo, está situado no grupo das estratégias de gerenciamento de recursos, com 1 das 4 habilidades, regulação de esforço.

3. Displicente - é o aluno que só visualiza o conteúdo no dia da entrega do exercício ou só visualiza o mesmo no momento em que está fazendo o exercício. Esse comportamento não se enquadra no modelo de cognição, pois o aluno, nesse caso está desenvolvendo suas respostas copiando diretamente das fontes, sem reflexão.

4. Ausente - é o aluno que quase não acessa o ColabWeb, geralmente só acessando quando aparece esporadicamente em alguma aula teórica presencial. Esse comportamento não se enquadra no modelo de cognição, pois o aluno, nesse caso está desenvolvendo suas respostas copiando diretamente das fontes, sem reflexão.

Em especial, foram considerados aspectos comportamentais encontrados nos registros dos logs do curso no ColabWeb para auxiliar na identificação dos perfis iniciais, ilustrados na Tabela 1 , quanto à resolução das atividades, visualização de conteúdo disponível e acesso à própria disciplina no ColabWeb. Como a disciplina foi ministrada para três cursos diferentes, foram formados clusters por curso para identificar esses perfis separados inicialmente.

\begin{tabular}{|l|c|c|c|c|}
\hline Log & Perfil Gerente & Perfil Inconsistente & Perfil Displicente & Perfil Ausente \\
\hline Resolução de atividade & $\begin{array}{c}\text { Fez todas antes da } \\
\text { data de entrega. }\end{array}$ & $\begin{array}{c}\text { Fez a maioria antes } \\
\text { da data de entrega. }\end{array}$ & $\begin{array}{c}\text { Fez todas na data de } \\
\text { entrega. }\end{array}$ & $\begin{array}{c}\text { Não fez a maioria } \\
\text { das atividades. }\end{array}$ \\
\hline Visualização do conteúdo & $\begin{array}{c}\text { Visualizou todo o } \\
\text { conteúdo } \\
\text { previamente e mais } \\
\text { de uma vez. }\end{array}$ & $\begin{array}{c}\text { Visualizou a maioria } \\
\text { do conteúdo } \\
\text { previamente e mais } \\
\text { de uma vez. }\end{array}$ & $\begin{array}{c}\text { Visualizou o } \\
\text { conteúdo apenas no da entrega das } \\
\text { diatividades. }\end{array}$ & $\begin{array}{c}\text { Visualizou o } \\
\text { conteúdo apenas } \\
\text { nas aulas teóricas. }\end{array}$ \\
\hline Acesso ao curso & $\begin{array}{c}\text { Acessou o curso mais } \\
\text { de uma vez em todas } \\
\text { as semanas de }\end{array}$ & $\begin{array}{c}\text { quase todas as } \\
\text { semanas de duração } \\
\text { duração do mesmo. }\end{array}$ & $\begin{array}{c}\text { Acesso o curso } \\
\text { somente no dia de } \\
\text { entrega de exercício. }\end{array}$ & $\begin{array}{c}\text { Acessou o curso } \\
\text { apenas em dias } \\
\text { de aulas teóricas. }\end{array}$ \\
\hline
\end{tabular}

Tabela 1. Critérios de classificação dos perfis cognitivos.

A Figura 2 representa os perfis cognitivos classificados por turma, a turma de Engenharia Civil que possui na sua totalidade 52 alunos cursando a disciplina. O perfil que mais se destaca é o "inconsistente" com 26 alunos identificados, que representa 50 $\%$ da turma, seguido do perfil ausente com 14 alunos, representando 26,92\% da turma, seguido do perfil Displicente com 9 alunos representando 17,30\% da turma e por último o perfil "gerente" com 3 aluno representando apenas 5,77\% da turma, na turma de Ciência Contábeis que possui 65 alunos matriculados. Nessa turma, o perfil que mais de destaca é o ausente, representando 43,07\% dos alunos, em segundo lugar o perfil "inconsistente" é reconhecido com $23,07 \%$ dos alunos, em terceiro lugar o perfil "displicente" com $18,46 \%$ e por último o perfil gerente representando $15,38 \%$ dos 
VII Congresso Brasileiro de Informática na Educação (CBIE 2018)

Anais do XXIX Simpósio Brasileiro de Informática na Educação (SBIE 2018)

alunos. E por fim na turma de Engenharia de Alimentos que possui 47 alunos matriculados, o perfil que mais se destaca é o "inconsistente" com 20 alunos identificados, representando 42,55\% da turma, seguido dos perfis "ausente" com 17 alunos e o "displicente" com 7 alunos, representando 36,17\% e 17,89 \% da turma respectivamente, e por último o perfil gerente com 3 alunos representa $6,38 \%$ da turma.

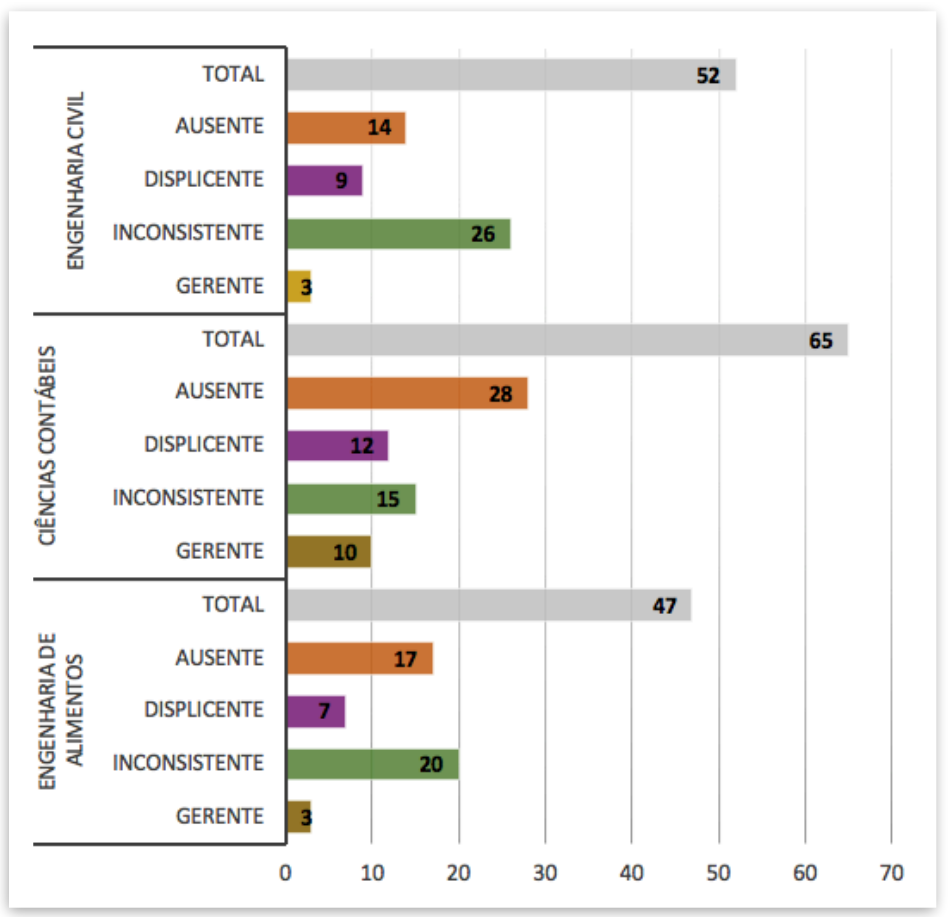

Figura 2. Perfis cognitivos separados por turma.

$\mathrm{Na}$ figura 3 estão descritos os perfis cognitivos de todas as turmas. Com 61 alunos o perfil "inconsistente", representando $37,19 \%$ dos alunos, é o mais comum da turma de 164 indivíduos, seguido dos perfis "ausente" com 59 alunos, representando 35,97\%, "displicente" com 28 alunos, representando 17,07\%, e por fim o perfil "gerente" com 16 alunos, representando $9,75 \%$ da turma.

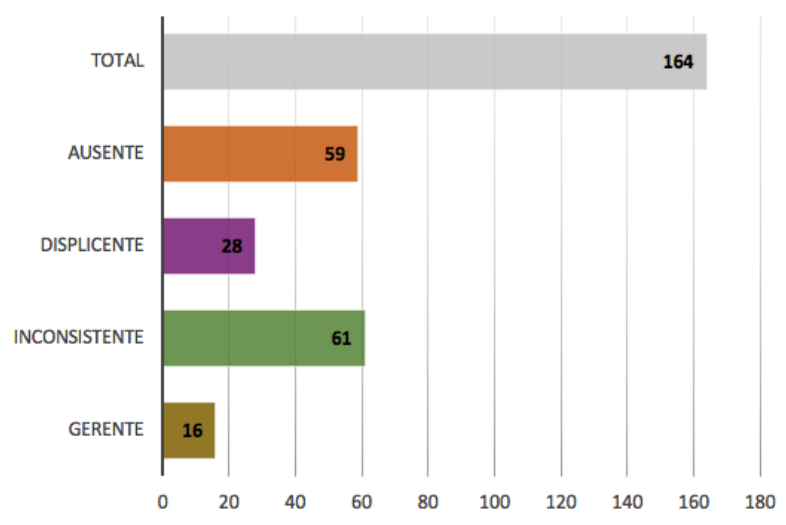

Figura 3. Perfis cognitivos de todos os cursos. 
VII Congresso Brasileiro de Informática na Educação (CBIE 2018)

Anais do XXIX Simpósio Brasileiro de Informática na Educação (SBIE 2018)

\section{Discussão}

Os perfis cognitivos encontrados podem servir em blended learning para adaptação de ambientes e conteúdos no AVA, como exemplo, no perfil "ausente". O conteúdo poderia ser adaptado para chamar mais atenção dos alunos ou o AVA sugerir outros tipos de conteúdo para o aluno como, vídeos, tutoriais e etc, após o primeiro mês de ter iniciado a disciplina.

Nesse artigo foi conduzido um estudo de caso exploratório para se definir esses perfis iniciais, retirados de 3 de 6 turmas da disciplina de Informática Instrumental do período acadêmico 2018/1. O objetivo era identificar esses perfis cognitivos iniciais e a partir deles fazer uma clusterização automática em todas as turmas do próximo semestre. Para isso, está em preparação a implementação de uma Decision Tree Classifier que classificará os alunos após um mês de disciplina, reavaliando-os a cada mês.

A vantagem de se usar um classificador automático é que o AVA pode associar certos indícios de comportamento e atuar imediatamente para prevenir que o aluno permaneça nele, como envio de mensagens de incentivo à realização das atividades, associação a conteúdo didático mais adequado àquele aluno ou uma sugestão de atividades de acordo com seus perfis atuais. Além disso, o algoritmo possibilita ao professor identificar quais alunos precisam de mais atenção, nesse caso, os alunos identificados nos perfis displicente e ausente seriam esses que requereriam mais atenção por tenderem a não completar as atividades. A partir disso o professor poderá tomar as decisões pedagógicas para tentar motivar mais o aluno.

Verificando os perfis cognitivos encontrados nos cursos de Engenharia Civil e Engenharia de Alimentos o perfil inconsistente se destaca. Analisando as duas turmas semanalmente durante as aulas práticas foi observado que as duas turmas eram somente de calouros, alunos que estão cursando o $1^{\circ}$. período de seus cursos. Os calouros geralmente são preocupados com o funcionamento do ensino superior, como as notas são calculadas, quantas disciplinas devem fazer entre outras situações que são novas para os mesmos, principalmente a gerência de tempo em disciplinas semipresenciais. Esse contexto de adaptações à nova realidade acadêmica pode ser um motivo preponderante para a grande quantidade de alunos dessas duas turmas, em especial, serem classificadas no perfil cognitivo inconsistente.

É importante salientar que os perfis displicentes e ausente estão mais atrelados a alunos que desistiram de cursar a disciplina e àqueles que entraram na universidade por meio de $2^{\mathrm{a}}$ ou $3^{\mathrm{a}}$ chamada de matrícula para a repescagem de alunos provenientes de ENEM e processo contínuo de avaliação do ensino médio, pois essas chamadas são realizadas até depois de um mês após o início do período letivo.

No curso de Ciências Contábeis o perfil que se destaca é o ausente. Analisando os alunos dessa turma verifica-se que a grande maioria dos alunos são veteranos e conforme os registros de matrícula do moodle vários deles são repetentes nessa disciplina. Talvez a situação ocorra, por esses alunos não enxergarem a importância da 
VII Congresso Brasileiro de Informática na Educação (CBIE 2018)

Anais do XXIX Simpósio Brasileiro de Informática na Educação (SBIE 2018)

disciplina para sua formação acadêmica. Como a disciplina é umas das obrigatórias em sua grade curricular a permanência na mesma é grande, porém com o decorrer do período o interesse vai diminuindo.

Outro ponto a ser evidenciado é que o perfil gerente no curso de Ciências Contábeis se sobressaiu quando comparado aos outros dois cursos. Como essa turma possui alunos veteranos e calouros não foi notado nenhuma característica especial que evidencie a grande quantidade de alunos no perfil gerente, porém é possível que com a mudança na metodologia da disciplina, exigindo mais interação entre os alunos, os alunos consigam gerenciar melhor o seu tempo.

\section{Considerações Finais}

Esse trabalho identifica perfis cognitivos iniciais encontrados a partir de variáveis comportamentais dos alunos em um AVA, na modalidade semipresencial (blended learning). A partir dessa análise foi possível definir quatro perfis cognitivos, dois deles enquadrando-se no modelo definido por Broadbent [2017] que são: gerente e o inconsistente. Os outros dois ainda são menos cognitivos e mais comportamentais puros, o displicente e o ausente.

A identificação desses perfis cognitivos iniciais ocorreu através da condução de um estudo de caso exploratório em uma disciplina básica de instrumentação em Informática ministrada para cursos de graduação na XXXXX. Através dos instrumentos de coleta utilizados foram identificados esses perfis e associadas atitudes que serão as sementes (seeds) na implementação de detecção automática de alunos nesses perfis para que sejam realizadas ações de prevenção pelo professor.

Inicialmente será implementada uma Decision Tree Classifier no AVA utilizado na disciplina para a identificação dos perfis cognitivos iniciais, relacionando a disponibilização de materiais e atividades diferentes a cada aluno, de acordo com o comportamento mais aderente a determinado perfil. Posteriormente, serão identificados perfis mais específicos, utilizando outras abordagens de clusterização automática para adaptar a interface inicial do curso no AVA para cada aluno, conforme seu perfil cognitivo temporário.

\section{Referências}

Aldhafeeri, F. M. (2015). Blended learning in higher education. International handbook of Elearning volume 2: Implementation and case studies, Vol. 2. (pp. 93), 93.

Allen, E. I., \& Seaman, J. (2013). Changing course: Ten years of tracking online education in the United States. Babson Survey Research Group.

Artino, A. R., \& Jones, K. (2012). Exploring the complex relations between achievement emotions and self-regulated learning behaviors in online learning. Internet and Higher Education, 15(3), 170-175.

Beaven, T., Codreanu, T., \& Creuze, A. (2014). Motivation in a language MOOC: Issues for course designers. In M. Elena, \& B. Elena (Eds.), Language MOOCs: 
VII Congresso Brasileiro de Informática na Educação (CBIE 2018)

Anais do XXIX Simpósio Brasileiro de Informática na Educação (SBIE 2018)

Providing learning, transcending boundaries (pp. 48e66). Berlin: De Gruyter Open.

Broadbent, J. (2017). Comparing online and blended learner's self-regulated learning strategies and academic performance. The Internet and Higher Education, 33, 24-32.

Kizilcec, R. F., Pérez-Sanagustín, M., ; Maldonado, J. J. (2017). Self-regulated learning strategies predict learner behavior and goal attainment in Massive Open Online Courses. Computers \& Education, 104, 18-33.

Kizilcec, R. F., Piech, C., \& Schneider, E. (2013, April). Deconstructing disengagement: Analyzing learner subpopulations in massive open online courses. In Proceedings of the third international conference on learning analytics and knowledge (pp. 170e179). ACM.

Moore, M. G.,; Kearsley, G. (2005). Distance education: A systems view (2nd ed.). Belmont, CA: Wadswort.

Picciano, Anthony G., Charles D. Dziuban e Charles R. Graham, eds (2013). Aprendizagem combinada: perspectivas de pesquisa . Vol. 2. Routledge.

Porter, Wendy W. (2014). " Blended learning in higher education: Institutional adoption and implementation".

Ratelle, C. F., Guay, F., Vallerand, R. J. and Larose, S., et al (2007) Autonomous, controlled, and amotivated types of academic motivation: A person-oriented analysis. Journal of Educational Psychology, Vol 99, No. 4, pp 734 - 746

Santos, M.; Oliveira, M. (2011). "Interação e Comunicação em Educação a Distância, I nteração e Comunicação em Comunidades de Aprendizagem", Santa Cruz-RN.

Silva, Viviane Gomes da; Bremgatner, Vitor; Ribeiro, Mirlem Rodrigues; Castro, Alberto; Nascimento, Rogério Patrício Chargas do. (2011): "Uma Experiência de "Virtualização" de Disciplina em Cursos de Graduação." Anais do XXII SBIE - XVII WIE.

Vallerand, R. J.; Pelletier, L. G. and Blais, M. R., et al (1992) The academic motivation scale: a measure of intrinsic, extrinsic, and amotivation in education. Educational and Psychological Measurement, pp 1003 - 1017.

Vanslambrouck, S., Zhu, C., Lombaerts, K., Philipsen, B., \& Tondeur, J. (2018). Motivação dos alunos e valor da tarefa subjetiva de participar em ambientes de aprendizagem on-line e mistos. A Internet e Ensino Superior , 36 , $33-40$.

Vanslambrouck, Silke; Tondeur, Jo e Zhu, Chang. (2015). Motivational Profiles of Adult Learners in Online and Blended Learning.

Watson, Sunnie Lee; Watson, William R.; Yu, Ji Hyun; Alamri, Hamdan e Mueller, Chad. (2017). "Learner profiles of attitudinal learning in a MOOC: An explanatory sequential mixed methods study".

Wigfield, A., \& Eccles, J. (2000). Expectancy-value theory of achievement motivation. Contemporary Educational Psychology, 25(1), 68-81. http://dx.doi.org/10.1006/ ceps. 1999.1015.

Yin R. K. (2014) "Estudo de caso: planejamento e métodos". 5a ed. Porto Alegre: Bookman. 